\title{
Hierarchical Value Maps of Smart Phones, Portal Sites, and Social Network Services Based on User Involvement
}

\author{
Sang Hee Kwon ${ }^{1}$, Min Kyung Cha ${ }^{1}$, Seo Young Lee ${ }^{2 *}$ \\ ${ }^{1}$ Sungkyunkwan University, Seoul, Korea \\ ${ }^{2}$ Yonsei University, Seoul, Korea \\ Email: "leeseoyoungann1004@gmail.com
}

Received 27 August 2015; accepted 26 September 2015; published 29 September 2015

Copyright (C) 2015 by authors and Scientific Research Publishing Inc.

This work is licensed under the Creative Commons Attribution International License (CC BY). http://creativecommons.org/licenses/by/4.0/

(c) (i) Open Access

\begin{abstract}
This study compares the hierarchical value maps (HVMs) for portal sites, smart phones, and social network services (SNSs) according to means-end chain theory. Means-end chain theory offers insights into how a media product satisfies the personal values of consumers. We derive the HVM for each media and investigate the effect of involvement on the HVM structure. A total of 424 individuals were queried for their opinions on the attributes, functional consequences, psychological consequences, and value of each media product through association pattern technique laddering. These elements constitute the value chain structure. Our findings show that the value of each media is determined by involvement levels. For each media, high-involvement user groups regard self-satisfaction as the most important value they aspire for. This study determines that different levels of involvement do not affect the relationships among the elements of the value chain. HVMs for smart phones, portal sites, and SNSs are focused on a special value. However, the level of involvement generally does not seem to alter the structures of HVMs.
\end{abstract}

\section{Keywords}

Value Chain Structure, Involvement, Smart Phone, Portal Site, Social Network Service

\section{Introduction}

In today's media-diverse world, technology has spawned a plethora of products and services, each with their own values and usage. Usage value has a large bearing over many aspects of a product or service. Moreover,

"Corresponding author.

How to cite this paper: Kwon, S. H., Cha, M. K., \& Lee, S. Y. (2015). Hierarchical Value Maps of Smart Phones, Portal Sites, and Social Network Services Based on User Involvement. Advances in Journalism and Communication, 3, 56-70. 
user needs evolve as the media environment evolves. Users tend to be financially self-sufficient and intellectually sophisticated; thus, their aspirations resonate with the high levels of Maslow's hierarchy of needs (Maslow, 1943; Baudrillard, 1970), such as desire to belong, respect, and self-actualization. New media creates shifts in the psychological needs of users along with their intentions for media use. Media usage will continue to evolve because technological advances enable easy access to content through different types of media at a low price.

We are interested in the selection of communication medium of users, who are influenced not only by the attributes of the medium but also by their psychological needs and values.

Given that user intention is the dominant motivation that drives media consumption, we must understand the motives, needs, and values of consumers. In particular, we should consider the services that media should provide as time passes and values change.

Consumer motives, needs, and values are particularly pertinent in the current media environment, where we see the proliferation of new media products, services, and functionality. Understanding consumer values provides media companies an advantage in the fiercely competitive consumer IT industry.

Christensen and Olson (2001) say that consumers prefer media services or functions involving consequences that are congruent with their personal values; in other words, the cognitive structures behind a consumers' personal values, and goals. This concept is the basis behind means-end chain theory, whereby the motivation of consumers in choosing a product is determined by the attributes and consequences that are in line with their personal values (Gutman, 1982; Peter \& Olson, 2005).

This study aims to understand the consumer decision-making process that underlies the value structure in the choice of using smart phones, portal sites, and social network services (SNSs). In addition, this study examines the effect of involvement on value structure.

We can observe rapid developments in media products and services and the tremendous enrichment of functionality. Understanding consumer values provides an advantage to media companies racing to stay ahead in the fiercely competitive consumer IT industry.

\section{Theoretical Background}

\section{Means and Chain Approach on Adoption of Smart Media}

Means-ends chain theory links a chain of attributes, consequences, and values to define the relationship between motives and goal states of consumers (Gutman, 1981). Attributes are the specific, concrete, and physical traits of a product. Consequences are the attributes of a product with perceived benefits. Value is linked to the abstract goals of an individual (ter Hofstede, Audenaert, Steenkamp, \& Wedel, 1998). Through the aforementioned relationship, we can construct a hierarchical value map (HVM) of the consumer decision-making process by considering not only the visual aspects of attributes but also the psychological, abstract, and symbolic steps to delve deep into the inner motives behind consumer behavior (see Table 1).

HVM provides a holistic framework to understand the choices made by media consumers. An HVM begins with product attributes and ends in personal values (Woodruff \& Gardial, 1996).

Involvement is defined as an individual's internal desire and interest for a product. It determines motivation, which may influence the goals that individuals strive for (Celsi \& Olson, 1988). Involvement causes a differing perception of products and individual values (Petty \& Cacioppo, 1986) because the matters that users consider important affect their motivations for information processing, attributes, and consequences. Differences in motivation affect the desires and satisfaction of individuals (Petty \& Cacioppo, 1986). Through this process, involvement affects attributes, consequences, and values, namely, the overall means-end chain structure.

\begin{tabular}{|c|c|c|c|}
\hline Level of Abstraction & 3 Steps & 6 Steps & 4 Steps \\
\hline \multirow[t]{2}{*}{ Abstract } & Value & $\begin{array}{l}\text { Terminal values } \\
\text { Instrumental values }\end{array}$ & Values \\
\hline & Consequences & $\begin{array}{l}\text { Psychological consequences } \\
\text { Functional consequences }\end{array}$ & $\begin{array}{l}\text { Psychological consequences } \\
\text { Functional consequences }\end{array}$ \\
\hline Concrete & Attributes & $\begin{array}{l}\text { Abstract attributes } \\
\text { Physical attributes }\end{array}$ & Attributes \\
\hline
\end{tabular}


In the present study, we investigate the supposition that involvement has an effect on any segment of HVM for media products. Accordingly, we propose the following research questions:

1) How does involvement affect media (smart phone, portal sites, and SNSs) users' perception of items in the four categories, namely, attributes, functional consequences, psychological consequences, and values?

2) Does involvement affect media (smart phones, portal sites, and SNSs) users' perception of the relationships among the categories, namely, attributes, functional consequences, psychological consequences, and values?

3) Does involvement change the HVM for users of smart phones, portal sites, and SNSs?

\section{Methodology}

\section{Laddering Interview Method}

Means-end chain theory was used to analyze the perception of users on smart phones, portal sites, and SNSs. User groups were queried on a list of items in each of the three categories (attributes, benefits, and values), relationships, and associations among the categories. The key variable involvement was used to assess if an influence exists on the value structure for media usage.

Consumers must understand how the attributes and benefits (consequences) of media relate to their inner values. To this end, researchers developed laddering as a method to investigate the minds of consumers. Laddering questionnaires can be either soft laddering or hard laddering (Grunert \& Grunert, 1995). Soft laddering involves one-on-one in-depth interview to assess the psychological disposition of respondents (Grunert \& Grunert, 1995). Hard laddering is a quantitative research method capable of overcoming the limitations of soft laddering. Numerous hard laddering techniques exist, but association pattern technique (APT) is the most sophisticated research method. In this study, we used APT laddering method to collect data for our means-end chain analysis.

First, we developed a list of attributes, functional consequences, psychological consequences, and values for measurements that are relevant for users of smart phones, portal sites, and SNSs. The items for these categories were extracted from a review of current literature. APT laddering was used to determine the relationships of items among categories (i.e., attributes-functional consequences, functional consequences-psychological consequences, and psychological consequences-value) for each media and establish a complete picture of the value structure. We also investigated the involvement factor for each media through a modified version of the Zaichkowsky personal involvement index (Zaichkowsky, 1985; Celsi \& Olsen, 1988; Petty \& Cacioppo, 1986; Huber, Beckmann, \& Hermann, 2004). We used SPSS 18.0 software and derived an average involvement score of 5.1773 for smart phones, 4.9008 for portal sites, and 4.3358 for SNSs. Media involvement scoring below the average score was categorized as low involvement. Conversely, media involvement scoring higher than the average was considered high involvement.

Frequency scoring is commonly used in APT laddering to extract the value structure from survey participants. Survey respondents marked in the implication matrix all the relationship pairs (e.g., attributes-functional consequences, psychological consequences-value) that are relevant to a consumer. The frequency score summarized the total number of times a relationship pair was marked by all survey respondents. However, the implication matrix can be quite complex and can be filled with unimportant relationships. To lessen the number of frequency scores, means-end chain theory only extracted the relevant relationships for the construction of the HVM diagram. Unimportant relationships were ignored.

Given that all important relationships (i.e., attributes, consequences, and values) for each item were extracted, we developed a comprehensive HVM summarizing the relationship between motivation and behavior (ladder).

\section{Research Results}

In this study, 424 individuals were surveyed about their use of smart phones, portal sites, and SNSs. The survey was conducted from April 2, 2014 to May 5, 2014. Most respondents were in their 20s (70.5\%). In total, 55.7\% were female and $44.3 \%$ were male. The most commonly used smart phone was Galaxy (49.1\% of respondents). In total, $54.7 \%$ of portal sites used Daum, and $93.9 \%$ of users used Kakao Talk.

\subsection{Results for Research Question 1}

Table 2 shows the frequencies of smart phone functional consequences. We could not determine any significant difference $\left(\chi^{2}=9.066, p=0.526\right)$ between high and low involvements through the chi-square test. This finding 
Table 2. Cross tabulation of functional consequence involvement for smart phones.

\begin{tabular}{|c|c|c|c|c|c|c|}
\hline \multirow{3}{*}{$\begin{array}{c}\text { Items } \\
\text { Daily life support }\end{array}$} & \multicolumn{4}{|c|}{ Involvement } & \multirow{2}{*}{\multicolumn{2}{|c|}{ Total }} \\
\hline & \multicolumn{2}{|c|}{ High Involvement } & \multicolumn{2}{|c|}{ Low Involvement } & & \\
\hline & 194 & $(11.8 \%)$ & 223 & $(12.2 \%)$ & 417 & $(12.0 \%)$ \\
\hline Self-expression & 136 & $(8.3 \%)$ & 139 & $(7.6 \%)$ & 275 & $(7.9 \%)$ \\
\hline Social relationship & 179 & $(10.9 \%)$ & 205 & $(11.2 \%)$ & 384 & $(11.1 \%)$ \\
\hline Mutual communication & 179 & $(10.9 \%)$ & 197 & $(10.8 \%)$ & 376 & $(10.8 \%)$ \\
\hline Speedy information retrieval/content acquisition & 174 & $(10.6 \%)$ & 194 & $(10.6 \%)$ & 368 & $(10.6 \%)$ \\
\hline Variety of information and content/acquisition & 175 & $(10.6 \%)$ & 198 & $(10.8 \%)$ & 373 & $(10.7 \%)$ \\
\hline Personal information and content storage & 162 & $(9.8 \%)$ & 179 & $(9.8 \%)$ & 341 & $(9.8 \%)$ \\
\hline Sharing information/content & 112 & $(6.8 \%)$ & 128 & $(7.0 \%)$ & 240 & $(6.9 \%)$ \\
\hline Work (mobile office) & 148 & $(9.0 \%)$ & 165 & $(9.0 \%)$ & 313 & $(9.0 \%)$ \\
\hline Entertainment & 186 & (11.3\%) & 199 & $(10.9 \%)$ & 385 & $(11.1 \%)$ \\
\hline Total & 1645 & $(100.0 \%)$ & 1827 & $(100.0 \%)$ & 3472 & $(100.0 \%)$ \\
\hline
\end{tabular}

$\chi^{2}=9.066, p=0.526$.

indicates that user perception of smart phone functional consequence is not dependent on the involvement factor.

Involvement affects user perception of psychological consequence for smart phone users (see Table 3). The results of the chi-square test showed no significant difference $\left(\chi^{2}=14.112, p=0.294\right)$ in the frequency of highand low-involvement user groups.

The most important smart phone values are shown in Table 4. Involvement has an influence on people's perception of value $\left(\chi^{2}=26.302, p<0.01\right)$.

The functional consequence involvement for portal sites (Table 5) indicates that the high-involvement group has a statistically significant difference with the low-involvement group $\left(\chi^{2}=26.656, p=0.003\right)$.

For portal site users, involvement does not affect psychological consequence items $\left(\chi^{2}=15.810, p=0.200\right.$; see Table 6).

Involvement levels have an effect on value items for portal site users (see Table $7 ; \chi^{2}=26.123, p=0.01$ ).

No statistical significance exists between high- and low-involvement SNS user groups for functional items $\left(\chi^{2}\right.$ $=12.451, p=0.256$; see Table 8 ).

The high-involvement user group has no statistically significant difference with the low-involvement user group for psychological consequence items of SNS users $\left(\chi^{2}=17.353, p=0.136\right.$; see Table 9$)$.

Finally, the important values pursued by SNS users are shown in Table 10. The high-involvement user group has a high statistically significant difference with the low-involvement user group $\left(\chi^{2}=35.834, p=0.000\right)$.

\subsection{Results for Question 2}

After the text edit has been completed, the paper is ready for the template. Duplicate the template file by using the Save As command, and use the naming convention prescribed by your journal for the name of your paper. In this newly created file, highlight all of the contents and import your prepared text file. You are now ready to style your paper.

\subsubsection{Impact of Involvement on the Means and Chains Relationships for Smart Phone User}

As shown in Table 11, the ranking of the most important relationships between attributes and functional consequences for smart phone users were very similar for both the high and low involvement groups; with the top three relationship for both groups being time/alarm-daily life support, calendar/diary/memo-daily life support and game-entertainment. 
Table 3. Cross tabulation of psychological consequence involvement for smart phones.

\begin{tabular}{cccccccc}
\hline & \multicolumn{3}{c}{ Involvement } & \multicolumn{2}{c}{ Total } \\
\cline { 2 - 5 } Item & \multicolumn{2}{c}{ High } & \multicolumn{2}{c}{ Low } & & $(11.6 \%)$ \\
Self satisfaction & 152 & $(10.8 \%)$ & 185 & $(12.3 \%)$ & 337 & $(4.3 \%)$ \\
Self pride & 64 & $(4.5 \%)$ & 60 & $(4.0 \%)$ & 124 & $(8.6 \%)$ \\
Self cultivation & 124 & $(8.8 \%)$ & 126 & $(8.4 \%)$ & 250 & $(5.5 \%)$ \\
Sincerity & 80 & $(5.7 \%)$ & 80 & $(5.3 \%)$ & 160 & $(50)$ \\
Individual personality & 117 & $(8.3 \%)$ & 114 & $(7.6 \%)$ & 231 & $(7.9 \%)$ \\
Desire to possess & 82 & $(5.8 \%)$ & 72 & $(4.8 \%)$ & 154 & $(5.3 \%)$ \\
Well-rounded social life & 174 & $(12.4 \%)$ & 195 & $(13.0 \%)$ & 369 & $(12.7 \%)$ \\
Convenience & 188 & $(13.4 \%)$ & 206 & $(13.7 \%)$ & 394 & $(13.6 \%)$ \\
Trust & 77 & $(5.5 \%)$ & 86 & $(5.7 \%)$ & 163 & $(5.6 \%)$ \\
Preference & 95 & $(6.8 \%)$ & 97 & $(6.5 \%)$ & 192 & $(6.6 \%)$ \\
Interest & 160 & $(11.4 \%)$ & 178 & $(11.9 \%)$ & 338 & $(11.6 \%)$ \\
Trendy/sophisticated & 94 & $(6.7 \%)$ & 101 & $(6.7 \%)$ & 195 & $(6.7 \%)$ \\
\hline
\end{tabular}

$\chi^{2}=14.112, p=0.294$.

Table 4. Cross tabulation of value and involvement for smart phones.

\begin{tabular}{|c|c|c|c|c|c|c|}
\hline \multirow{3}{*}{$\begin{array}{c}\text { Item } \\
\text { Self-realization }\end{array}$} & \multicolumn{4}{|c|}{ Involvement } & \multirow{2}{*}{\multicolumn{2}{|c|}{ Total }} \\
\hline & \multicolumn{2}{|c|}{ High } & \multicolumn{2}{|c|}{ Low } & & \\
\hline & 162 & $(9.6 \%)$ & 174 & $(9.5 \%)$ & 336 & $(9.6 \%)$ \\
\hline Vibrant life & 157 & $(9.3 \%)$ & 188 & $(10.3 \%)$ & 345 & $(9.8 \%)$ \\
\hline Self-satisfaction & 180 & $(10.7 \%)$ & 190 & $(10.4 \%)$ & 370 & $(10.5 \%)$ \\
\hline Self-respect & 133 & $(7.9 \%)$ & 157 & $(8.6 \%)$ & 290 & $(8.3 \%)$ \\
\hline Sense of belonging & 116 & $(6.9 \%)$ & 140 & $(7.6 \%)$ & 256 & $(7.3 \%)$ \\
\hline Being well-respected & 134 & $(8.0 \%)$ & 118 & $(6.4 \%)$ & 252 & $(7.2 \%)$ \\
\hline Fun and enjoyment & 164 & $(9.8 \%)$ & 189 & $(10.3 \%)$ & 353 & $(10.0 \%)$ \\
\hline Warm relationship with others & 167 & $(9.9 \%)$ & 184 & $(10.0 \%)$ & 351 & $(10.0 \%)$ \\
\hline Empowerment & 125 & $(7.4 \%)$ & 134 & $(7.3 \%)$ & 259 & $(7.4 \%)$ \\
\hline Happiness & 162 & $(9.6 \%)$ & 163 & $(8.9 \%)$ & 325 & $(9.3 \%)$ \\
\hline Inner Balance & 111 & $(6.6 \%)$ & 101 & (5.5\%) & 212 & $(6.0 \%)$ \\
\hline Aesthetic aspect & 71 & $(4.2 \%)$ & 93 & (5.1\%) & 164 & $(4.7 \%)$ \\
\hline Total & 1682 & $(100.0 \%)$ & 1831 & $(100.0 \%)$ & 3513 & $(100.0 \%)$ \\
\hline
\end{tabular}

$\chi^{2}=26,302, p<0.01$.

Table 5. Cross tabulation of functional consequence and involvement for portal sites.

\begin{tabular}{cccccc}
\hline \multirow{2}{*}{ Item } & \multicolumn{3}{c}{ Involvement } & \multicolumn{2}{c}{ Total } \\
\cline { 2 - 5 } & \multicolumn{2}{c}{ High } & \multicolumn{2}{c}{ Low } & \\
Daily life support & 188 & $(11.6 \%)$ & 205 & $(12.6 \%)$ & $393(12.1 \%)$ \\
Self-expression & 104 & $(6.4 \%)$ & 109 & $(6.7 \%)$ & $213(6.5 \%)$ \\
Social relationship & 131 & $(8.1 \%)$ & 130 & $(8.0 \%)$ & $261(8.0 \%)$ \\
Mutual communication & 136 & $(8.4 \%)$ & 132 & $(8.1 \%)$ & $268(8.2 \%)$ \\
Speedy information retrieval/content acquisition & 191 & $(11.8 \%)$ & 194 & $(11.9 \%)$ & $385(11.8 \%)$ \\
Variety of information and content/acquisition & 187 & $(11.5 \%)$ & 194 & $(11.9 \%)$ & $381(11.7 \%)$ \\
Personal information and content storage & 170 & $(10.5 \%)$ & 172 & $(10.6 \%)$ & $342(10.5 \%)$ \\
Sharing information/content & 163 & $(10.0 \%)$ & 147 & $(9.0 \%)$ & $310(9.5 \%)$ \\
Work (mobile office) & 168 & $(10.3 \%)$ & 152 & $(9.3 \%)$ & $320(9.8 \%)$ \\
Entertainment & 187 & $(11.5 \%)$ & 192 & $(11.8 \%)$ & $379(11.7 \%)$ \\
Total & 1625 & $(100.0 \%)$ & 1627 & $(100.0 \%)$ & $3252(100.0 \%)$ \\
\hline
\end{tabular}

$\chi^{2}=26.656, p=0.003$ 
Table 6. Cross tabulation of psychological consequence and involvement for portal site users.

\begin{tabular}{|c|c|c|c|c|c|c|}
\hline \multirow{3}{*}{$\begin{array}{c}\text { Items } \\
\text { Self-satisfaction }\end{array}$} & \multicolumn{4}{|c|}{ Involvement } & \multirow{2}{*}{\multicolumn{2}{|c|}{ Total }} \\
\hline & \multicolumn{2}{|c|}{ High } & \multicolumn{2}{|c|}{ Low } & & \\
\hline & 152 & $(14.5 \%)$ & 158 & $(15.0 \%)$ & 307 & $(14.8 \%)$ \\
\hline Self-pride & 64 & $(6.8 \%)$ & 56 & $(5.3 \%)$ & 126 & $(6.1 \%)$ \\
\hline Self-cultivation & 124 & $(11.3 \%)$ & 105 & $(10.0 \%)$ & 221 & $(10.6 \%)$ \\
\hline Sincerity & 80 & $(7.0 \%)$ & 74 & $(7.0 \%)$ & 146 & $(7.0 \%)$ \\
\hline Individual personality & 117 & $(10.2 \%)$ & 93 & $(8.9 \%)$ & 198 & $(9.5 \%)$ \\
\hline Desire to possess & 82 & $(7.6 \%)$ & 72 & $(6.9 \%)$ & 150 & $(7.2 \%)$ \\
\hline Well-rounded social life & 174 & $(17.3 \%)$ & 191 & $(18.2 \%)$ & 368 & $(17.7 \%)$ \\
\hline Convenience & 188 & $(18.4 \%)$ & 204 & $(19.4 \%)$ & 393 & $(18.9 \%)$ \\
\hline Trust & 77 & $(8.5 \%)$ & 88 & $(8.4 \%)$ & 175 & $(8.4 \%)$ \\
\hline Preference & 95 & $(8.6 \%)$ & 102 & $(9.7 \%)$ & 190 & $(9.2 \%)$ \\
\hline Interest & 160 & $(16.3 \%)$ & 166 & $(15.8 \%)$ & 333 & $(16.0 \%)$ \\
\hline Trendy/sophisticated & 94 & $(8.3 \%)$ & 79 & (7.5\%) & 164 & $(7.9 \%)$ \\
\hline Total & 1026 & $(100.0 \%)$ & 1050 & $(100.0 \%)$ & 2076 & (100.0\%) \\
\hline
\end{tabular}

$\chi^{2}=15.810, p=0.200$.

Table 7. Cross tabulation of value and involvement for portal sites users.

\begin{tabular}{ccccccc}
\hline \multirow{2}{*}{ Items } & \multicolumn{3}{c}{ Involvement } & \multicolumn{2}{c}{ Total } \\
\cline { 2 - 5 } & \multicolumn{2}{c}{ High } & \multicolumn{2}{c}{ Low } & & $(10.1 \%)$ \\
Self-realisation & 162 & $(9.8 \%)$ & 172 & $(10.4 \%)$ & 334 & $(10.2 \%)$ \\
Vibrant life & 166 & $(10.0 \%)$ & 171 & $(10.3 \%)$ & 337 & $(10.4 \%)$ \\
Sense of accomplishment & 177 & $(10.7 \%)$ & 168 & $(10.1 \%)$ & 345 & $(8.5 \%)$ \\
Self-respect & 138 & $(8.3 \%)$ & 144 & $(8.7 \%)$ & 282 & $(7.2 \%)$ \\
Sense of belonging & 122 & $(7.3 \%)$ & 116 & $(7.0 \%)$ & 238 & $(7.1 \%)$ \\
Being well-respected & 120 & $(7.2 \%)$ & 116 & $(7.0 \%)$ & 236 & $(10.2 \%)$ \\
Fun and enjoyment & 176 & $(10.6 \%)$ & 163 & $(9.8 \%)$ & 339 & $(9.9 \%)$ \\
Warm relationship with others & 162 & $(9.8 \%)$ & 166 & $(10.0 \%)$ & 328 & $(7.1 \%)$ \\
Empowerment & 123 & $(7.4 \%)$ & 113 & $(6.8 \%)$ & 236 & $(9.5 \%)$ \\
Happiness & 158 & $(9.5 \%)$ & 156 & $(9.4 \%)$ & 314 & $(9.5 \%)$ \\
Inner balance & 93 & $(5.6 \%)$ & 95 & $(5.7 \%)$ & 188 & $(5.7 \%)$ \\
Aesthetic aspect & 63 & $(3.8 \%)$ & 77 & $(4.6 \%)$ & 140 & $(4.2 \%)$ \\
Total & 1660 & $(100.0 \%)$ & 1657 & $(100.0 \%)$ & 3317 & $(100.0 \%)$ \\
\hline
\end{tabular}

$\chi^{2}=26.123, p=0.01$.

Table 8. Cross tabulation of functional consequence and involvement for SNS users.

\begin{tabular}{ccccccc}
\hline \multirow{2}{*}{ Items } & \multicolumn{3}{c}{ Involvement } & \multicolumn{2}{c}{ Total } \\
\cline { 2 - 6 } & \multicolumn{2}{c}{ High } & \multicolumn{3}{c}{ Low } & \\
Daily life support & 199 & $(13.0 \%)$ & 198 & $(13.3 \%)$ & 397 & $(13.2 \%)$ \\
Self-expression & 154 & $(10.0 \%)$ & 146 & $(9.8 \%)$ & 300 & $(9.9 \%)$ \\
Social relationship & 106 & $(6.9 \%)$ & 112 & $(7.5 \%)$ & 218 & $(7.2 \%)$ \\
Mutual communication & 207 & $(13.5 \%)$ & 203 & $(13.7 \%)$ & 410 & $(13.6 \%)$ \\
Speedy information retrieval/content acquisition & 183 & $(11.9 \%)$ & 173 & $(11.7 \%)$ & 356 & $(11.8 \%)$ \\
Variety of information and content/acquisition & 147 & $(9.6 \%)$ & 151 & $(10.2 \%)$ & 298 & $(9.9 \%)$ \\
Personal information and content storage & 149 & $(9.7 \%)$ & 124 & $(8.4 \%)$ & 273 & $(9.0 \%)$ \\
Sharing information/content & 73 & $(4.8 \%)$ & 77 & $(5.2 \%)$ & 150 & $(5.0 \%)$ \\
Work (mobile office) & 137 & $(8.9 \%)$ & 126 & $(8.5 \%)$ & 263 & $(8.7 \%)$ \\
Entertainment & 179 & $(11.7 \%)$ & 174 & $(11.7 \%)$ & 353 & $(11.7 \%)$ \\
Total & 1534 & $(100.0 \%)$ & 1484 & $(100.0 \%)$ & 3018 & $(100.0 \%)$ \\
\hline
\end{tabular}

$\chi^{2}=12.451, p=0.256$. 
Table 9. Cross tabulation of psychological consequence and involvement for SNS users.

\begin{tabular}{cccccccc}
\hline \multirow{2}{*}{ Items } & \multicolumn{3}{c}{ Involvement } & \multicolumn{2}{c}{ Total } \\
\cline { 2 - 5 } & \multicolumn{2}{c}{ High } & \multicolumn{2}{c}{ Low } & & $(11.4 \%)$ \\
Self-satisfaction & 162 & $(11.0 \%)$ & 163 & $(11.9 \%)$ & 325 & $(4.3 \%)$ \\
Self-pride & 64 & $(4.3 \%)$ & 59 & $(4.3 \%)$ & 123 & $(7.1 \%)$ \\
Self-cultivation & 104 & $(7.1 \%)$ & 99 & $(7.2 \%)$ & 203 & $(8.8 \%)$ \\
Sincerity & 128 & $(8.7 \%)$ & 122 & $(8.9 \%)$ & 250 & $(5.1 \%)$ \\
Individual personality & 80 & $(5.4 \%)$ & 64 & $(4.7 \%)$ & 144 & $(1 \%)$ & $(12.4 \%)$ \\
Desire to possess & 183 & $(12.4 \%)$ & 171 & $(12.5 \%)$ & 354 & $(10.9 \%)$ \\
Well-rounded social life & 164 & $(11.1 \%)$ & 147 & $(10.7 \%)$ & 311 & $(12.8 \%)$ \\
Convenience & 182 & $(12.3 \%)$ & 181 & $(13.2 \%)$ & 363 & $(12.8 \%)$ \\
Trust & 69 & $(4.7 \%)$ & 54 & $(3.9 \%)$ & 123 & $(4.3 \%)$ \\
Preference & 85 & $(5.8 \%)$ & 81 & $(5.9 \%)$ & 166 & $(5.8 \%)$ \\
Interest & 174 & $(11.8 \%)$ & 163 & $(11.9 \%)$ & 337 & $(11.8 \%)$ \\
Trendy/sophisticated & 79 & $(5.4 \%)$ & 66 & $(4.8 \%)$ & 145 & $(5.1 \%)$ \\
Total & 1474 & $(100.0 \%)$ & 1370 & $(100.0 \%)$ & 2844 & $(100.0 \%)$ \\
\hline
\end{tabular}

$\chi^{2}=17.353, p=0.136$

Table 10. Cross tabulation of value and involvement for SNS users.

\begin{tabular}{|c|c|c|c|c|c|c|}
\hline \multirow{3}{*}{$\begin{array}{c}\text { Items } \\
\text { Self-realisation }\end{array}$} & \multicolumn{4}{|c|}{ Involvement } & \multirow{2}{*}{\multicolumn{2}{|c|}{ Total }} \\
\hline & \multicolumn{2}{|c|}{ High } & \multicolumn{2}{|c|}{ Low } & & \\
\hline & 149 & $(8.9 \%)$ & 158 & $(9.8 \%)$ & 307 & $(9.3 \%)$ \\
\hline Vibrant life & 174 & $(10.4 \%)$ & 157 & $(9.8 \%)$ & 331 & $(10.1 \%)$ \\
\hline Sense of accomplishment & 182 & $(10.9 \%)$ & 163 & $(10.1 \%)$ & 345 & $(10.5 \%)$ \\
\hline Self-respect & 138 & $(8.2 \%)$ & 135 & $(8.4 \%)$ & 273 & $(8.3 \%)$ \\
\hline Sense of belonging & 141 & $(8.4 \%)$ & 125 & $(7.8 \%)$ & 266 & $(8.1 \%)$ \\
\hline Being well-respected & 135 & $(8.1 \%)$ & 120 & $(7.5 \%)$ & 255 & $(7.8 \%)$ \\
\hline Fun and enjoyment & 173 & $(10.3 \%)$ & 170 & $(10.6 \%)$ & 343 & $(10.4 \%)$ \\
\hline Warm relationship with others & 180 & $(10.7 \%)$ & 177 & $(11.0 \%)$ & 357 & $(10.9 \%)$ \\
\hline Empowerment & 106 & $(6.3 \%)$ & 101 & $(6.3 \%)$ & 207 & $(6.3 \%)$ \\
\hline Happiness & 167 & $(10.0 \%)$ & 139 & $(8.6 \%)$ & 306 & (9.3\%) \\
\hline Inner balance & 80 & $(4.8 \%)$ & 88 & $(5.5 \%)$ & 168 & $(5.1 \%)$ \\
\hline Aesthetic aspect & 50 & $(3.0 \%)$ & 77 & $(4.8 \%)$ & 127 & $(3.9 \%)$ \\
\hline Total & 1675 & (100.0\%) & 1610 & (100.0\%) & 3285 & (100.0\%) \\
\hline
\end{tabular}

$\chi^{2}=35.834, p=0.000$

Table 11. Implication matrix of relationships between attributes and functional consequences for smart phone users by involvement.

\begin{tabular}{cccccccc}
\hline \multirow{2}{*}{ Items } & \multicolumn{4}{c}{ Involvement } & \multicolumn{3}{c}{ Total } \\
\cline { 2 - 5 } & \multicolumn{3}{c}{ High } & \multicolumn{2}{c}{ Low } & & \\
Time/alarm-daily life support & 183 & $(12.2 \%)$ & 222 & $(13.1 \%)$ & 405 & $(12.7 \%)$ \\
Calendar/diary/memo-daily supp life support & 172 & $(11.5 \%)$ & 200 & $(11.8 \%)$ & 372 & $(11.7 \%)$ \\
Game-entertainment & 168 & $(11.2 \%)$ & 188 & $(11.1 \%)$ & 356 & $(11.2 \%)$ \\
Maps/navigation-daily life support & 151 & $(10.1 \%)$ & 177 & $(10.5 \%)$ & 328 & $(10.3 \%)$ \\
Electronic commerce-daily life support & 147 & $(9.8 \%)$ & 154 & $(9.1 \%)$ & 301 & $(9.5 \%)$ \\
Internet surfing-speedy information retrieval/contents acquisition & 137 & $(9.2 \%)$ & 150 & $(8.9 \%)$ & 287 & $(9.0 \%)$ \\
Internet surfing-variety of information/content acquisition & 137 & $(9.2 \%)$ & 137 & $(8.1 \%)$ & 274 & $(8.6 \%)$ \\
Voice call/video call-social relationship & 135 & $(9.0 \%)$ & 141 & $(8.3 \%)$ & 276 & $(8.7 \%)$ \\
SNS use-social relationship & 134 & $(9.0 \%)$ & 172 & $(10.2 \%)$ & 306 & $(9.6 \%)$ \\
Voice call-telephone call/mutual communication & 132 & $(8.8 \%)$ & 148 & $(8.8 \%)$ & 280 & $(8.8 \%)$ \\
Total & 1496 & $(100.0 \%)$ & 1689 & $(100.0 \%)$ & 3185 & $(100.0 \%)$ \\
& & & & & &
\end{tabular}

$\chi^{2}=35.834, p=0.000$ 
As shown in Table 12, the top three functional consequence-psychological consequence relationships for high and low involvement groups are entertainment-fun, followed by mutual communication-Well-rounded social life and social Relationship-Well-rounded social life. There was very little difference in how the high and low involvement groups rated importance of the lesser ranked relationships.

As shown in Table 13, the top three psychological consequences relationships for both high and low users are well-rounded social life-warm relationship with others, interest-Fun and enjoyment and Preference (taste)-happiness. There was very little difference between how the high and low involvement groups ranked the other 7 relationships.

Table 12. Implication matrix of relationships between function consequences and psychological consequences for smart phone users by involvement.

\begin{tabular}{|c|c|c|c|c|c|c|}
\hline \multirow{3}{*}{$\begin{array}{c}\text { Items } \\
\text { Entertainment-fun }\end{array}$} & \multicolumn{4}{|c|}{ Involvement } & \multirow{2}{*}{\multicolumn{2}{|c|}{ Total }} \\
\hline & \multicolumn{2}{|c|}{ High } & \multicolumn{2}{|c|}{ Low } & & \\
\hline & 134 & $(12.0 \%)$ & 151 & $(11.4 \%)$ & 285 & $(11.7 \%)$ \\
\hline Mutual communication-well-rounded social life & 129 & $(11.5 \%)$ & 149 & $(11.2 \%)$ & 278 & $(11.4 \%)$ \\
\hline Social Relationship-well-rounded social life & 127 & $(11.4 \%)$ & 149 & $(11.2 \%)$ & 276 & $(11.3 \%)$ \\
\hline Work (mobile office)-convenience & 123 & $(11.0 \%)$ & 123 & $(9.3 \%)$ & 246 & $(10.1 \%)$ \\
\hline Daily life support-convenience & 115 & $(10.3 \%)$ & 139 & $(10.5 \%)$ & 254 & $(10.4 \%)$ \\
\hline Speed information retrieval/content acquisition-convenience & 109 & $(9.7 \%)$ & 131 & $(9.9 \%)$ & 240 & $(9.8 \%)$ \\
\hline Personal information content storage-convenience & 99 & $(8.9 \%)$ & 117 & $(8.8 \%)$ & 216 & $(8.8 \%)$ \\
\hline Variety of information/content acquisition-convenience & 98 & $(8.8 \%)$ & 133 & $(10.0 \%)$ & 231 & $(9.5 \%)$ \\
\hline Self-expression-self satisfaction & 95 & $(8.5 \%)$ & 127 & $(9.6 \%)$ & 222 & $(9.1 \%)$ \\
\hline Sharing info/contents-convenience & 89 & $(8.0 \%)$ & 107 & $(8.1 \%)$ & 196 & $(8.0 \%)$ \\
\hline Total & 1118 & $(100.0 \%)$ & 1326 & $(100.0 \%)$ & 2444 & $(100.0 \%)$ \\
\hline
\end{tabular}

Table 13. Implication matrix of relationships between psychological consequences and value for smart phone users by involvement.

\begin{tabular}{|c|c|c|c|c|c|c|}
\hline \multirow{2}{*}{ Items } & \multicolumn{4}{|c|}{ Involvement } & \multirow{2}{*}{\multicolumn{2}{|c|}{ Total }} \\
\hline & \multicolumn{2}{|c|}{ High } & \multicolumn{2}{|c|}{ Low } & & \\
\hline Well-rounded social life-warm relationship with others & 111 & $(14.2 \%)$ & 132 & $(15.5 \%)$ & 243 & $(14.9 \%)$ \\
\hline interest-fun and enjoyment & 95 & $(12.2 \%)$ & 110 & $(12.9 \%)$ & 205 & $(12.6 \%)$ \\
\hline Preference (taste)-happiness & 81 & $(10.4 \%)$ & 83 & $(9.8 \%)$ & 164 & $(10.1 \%)$ \\
\hline interest-happiness & 85 & $(10.9 \%)$ & 73 & $(8.6 \%)$ & 158 & $(9.7 \%)$ \\
\hline Self-cultivation-self accomplishment & 87 & $(11.2 \%)$ & 70 & $(8.2 \%)$ & 157 & $(9.6 \%)$ \\
\hline Trust-warm relationship with others & 65 & $(8.3 \%)$ & 81 & $(9.5 \%)$ & 146 & $(9.0 \%)$ \\
\hline Preference/taste-fun and enjoyment & 72 & $(9.2 \%)$ & 73 & $(8.6 \%)$ & 145 & $(8.9 \%)$ \\
\hline Self-cultivation-self-realisation & 62 & $(7.9 \%)$ & 83 & $(9.8 \%)$ & 145 & $(8.9 \%)$ \\
\hline Self-pride-self accomplishment & 62 & $(7.9 \%)$ & 76 & $(8.9 \%)$ & 138 & $(8.5 \%)$ \\
\hline Convenience-vibrant life & 60 & $(7.7 \%)$ & 69 & $(8.1 \%)$ & 129 & $(7.9 \%)$ \\
\hline Total & 780 & $(100.0 \%)$ & 850 & $(100.0 \%)$ & 1630 & $(100.0 \%)$ \\
\hline
\end{tabular}




\subsubsection{Impact of Involvement on the Means and Chains Relationships for Portal Site Users}

As shown in Table 14, the three most important attributes to functional consequences relationships for portal sites for both high and low involvement groups are game-daily life support, maps-daily life support followed by multimedia content-entertainment. There were differences in how the low and high involvement groups rated the lower rank items.

As shown in Table 15, the three most important relationships between psychological consequences and functional consequences for the high involvement group are work (mobile office)-Convenience, social relationship-vibrant life followed by mutual communication-vibrant life. For the low involvement group, the most important relationships are social relationship-vibrant life, mutual communication-vibrant life followed by daily life support-convenience.

As shown in Table 16, the three most important relationships between psychological consequences and values were the same for both the high and low involvement groups; which were pursuing personal characterwarm relationship with others, interest-fun and enjoyment followed by self-satisfaction-self realization.

Table 14. Implication matrix of relationships of attributes and functional consequences for portal site users by involvement.

\begin{tabular}{|c|c|c|c|c|c|c|}
\hline \multirow{2}{*}{ Items } & \multicolumn{4}{|c|}{ Involvement } & \multirow{2}{*}{\multicolumn{2}{|c|}{ Total }} \\
\hline & \multicolumn{2}{|c|}{ High } & \multicolumn{2}{|c|}{ Low } & & \\
\hline Internet surfing-daily life support & 96 & $(5.9 \%)$ & 117 & $(7.1 \%)$ & 213 & $(6.5 \%)$ \\
\hline Internet surfing-seedy information retrieval/content acquisition & 124 & $(7.7 \%)$ & 125 & $(7.6 \%)$ & 249 & $(7.6 \%)$ \\
\hline Cloud-Information/content-personal storage & 125 & $(7.7 \%)$ & 114 & $(7.0 \%)$ & 239 & $(7.3 \%)$ \\
\hline News/life information-speedy information retrieval/content acquisition. & 127 & $(7.9 \%)$ & 128 & $(7.8 \%)$ & 255 & $(7.8 \%)$ \\
\hline News/life information—a variety of information/content acquisition & 118 & $(7.3 \%)$ & 108 & $(6.6 \%)$ & 226 & $(6.9 \%)$ \\
\hline Knowledge dictionary/variety of information/content acquisition & 109 & $(6.7 \%)$ & 110 & $(6.7 \%)$ & 219 & $(6.7 \%)$ \\
\hline Multimedia content-entertainment & 130 & $(8.1 \%)$ & 132 & $(8.1 \%)$ & 262 & $(8.1 \%)$ \\
\hline Game-daily life support & 145 & $(9.0 \%)$ & 148 & $(9.1 \%)$ & 293 & $(9.1 \%)$ \\
\hline Maps-daily life support & 145 & $(9.0 \%)$ & 148 & $(9.1 \%)$ & 293 & $(9.1 \%)$ \\
\hline e-commerce/daily life support & 127 & $(7.9 \%)$ & 143 & $(8.8 \%)$ & 270 & $(8.3 \%)$ \\
\hline Total & 1608 & $(100.0 \%)$ & 1629 & $(100.0 \%)$ & 3237 & $(100.0 \%)$ \\
\hline
\end{tabular}

Table 15. Implication matrix of relationships of psychological consequences and functional consequences for portal site users by involvement.

\begin{tabular}{ccccccc}
\hline \multirow{2}{*}{ Items } & \multicolumn{3}{c}{ Involvement } & \multicolumn{3}{c}{ Total } \\
\cline { 2 - 5 } Daily life support-convenience & \multicolumn{2}{c}{ High } & \multicolumn{1}{c}{ Low } & & \\
Self-expression-self satisfaction & 106 & $(10.3 \%)$ & 139 & $(12.3 \%)$ & 245 & $(11.3 \%)$ \\
Social relationship-vibrant life & 95 & $(9.2 \%)$ & 103 & $(9.1 \%)$ & 198 & $(9.2 \%)$ \\
Mutual communication-vibrant life & 125 & $(12.2 \%)$ & 145 & $(12.8 \%)$ & 270 & $(12.5 \%)$ \\
Speedy information retrieval/content acquisition-convenience & 120 & $(11.7 \%)$ & 134 & $(11.8 \%)$ & 254 & $(11.8 \%)$ \\
Variety of information/content acquisition-convenience & 115 & $(11.2 \%)$ & 119 & $(10.5 \%)$ & 234 & $(10.8 \%)$ \\
Information content storage-convenience & 110 & $(10.7 \%)$ & 119 & $(10.5 \%)$ & 229 & $(10.6 \%)$ \\
Sharing info/contents-convenience & 101 & $(9.8 \%)$ & 108 & $(9.5 \%)$ & 209 & $(9.7 \%)$ \\
Work (mobile office)-convenience & 131 & $(12.7 \%)$ & 124 & $(11.0 \%)$ & 255 & $(11.8 \%)$ \\
Total & 1028 & $(100.0 \%)$ & 1132 & $(100.0 \%)$ & 2160 & $(100.0 \%)$ \\
\hline
\end{tabular}


Table 16. Implication matrix of relationships of psychological consequences and values for portal site users by involvement

\begin{tabular}{|c|c|c|c|c|c|c|}
\hline \multirow{2}{*}{ Items } & \multicolumn{4}{|c|}{ Involvement } & \multirow{2}{*}{\multicolumn{2}{|c|}{ Total }} \\
\hline & \multicolumn{2}{|c|}{ High } & \multicolumn{2}{|c|}{ Low } & & \\
\hline Self-satisfaction-self-realisation & 91 & $(10.8 \%)$ & 93 & $(11.7 \%)$ & 184 & $(11.3 \%)$ \\
\hline Self-pride-self accomplishment & 78 & $(9.3 \%)$ & 65 & $(8.2 \%)$ & 143 & $(8.8 \%)$ \\
\hline Self-cultivation-self accomplishment & 82 & $(9.8 \%)$ & 65 & $(8.2 \%)$ & 147 & $(9.0 \%)$ \\
\hline Integrity-self accomplishment & 59 & $(7.0 \%)$ & 69 & $(8.7 \%)$ & 128 & $(7.8 \%)$ \\
\hline Pursuing personal character-warm relationship with others & 112 & $(13.3 \%)$ & 127 & $(16.0 \%)$ & 239 & $(14.6 \%)$ \\
\hline Desire to possess-vibrant life & 66 & $(7.9 \%)$ & 75 & $(9.5 \%)$ & 141 & $(8.6 \%)$ \\
\hline Well-rounded social life-warm relationship with others & 65 & $(7.7 \%)$ & 74 & $(9.3 \%)$ & 139 & $(8.5 \%)$ \\
\hline Preference taste-fun and enjoyment & 80 & $(9.5 \%)$ & 69 & $(8.7 \%)$ & 149 & $(9.1 \%)$ \\
\hline Interest-fun and enjoyment & 116 & $(13.8 \%)$ & 97 & $(12.2 \%)$ & 213 & $(13.1 \%)$ \\
\hline Interest-happiness & 90 & $(10.7 \%)$ & 59 & $(7.4 \%)$ & 149 & $(9.1 \%)$ \\
\hline Total & 839 & $(100.0 \%)$ & 793 & $(100.0 \%)$ & 1632 & (100.0\%) \\
\hline
\end{tabular}

\subsubsection{Impact of Involvement on the Means and Chains Relationships for SNS Users}

For SNS users, Table 17 shows the importance rankings of the relationships between the attributes and functional consequences were the exactly the same for both the high and low involvement groups. The three most important relationships were my account/scheduling-daily life support, online social networking-social relationships and friends/institution introduction-social relationships.

Table 18 shows the two most important relationships between psychological consequences and functional consequences for bothe the high and low involvement groups were mutual communication-well-round social life followed by a variety of information/content acquisition-convenience. Minor differences appeared in the ranking of lesser important items.

Table 19 shows the three most important relationships between psychological consequences and value for both the low and high involvement groups are Well-rounded social life-Friendly relationship with others, desire to possess-warm relationship with others followed by interest-fun and enjoyment. Minor differences appeared in the ranking of lesser important items.

\subsection{Results for Research Question 3}

Question 3 aims to construct HVMs through means-end chain theory and to connect the relationships among the categories (attributes, functional consequences, psychological consequences, and value) of smart phones, portal sites, and SNSs. The results for Question 3 is used to generate goal hierarchy for using smart media and incorporates the results obtained in Questions 1 and 2.

The HVM for high-involvement smart phones users is shown in Figure 1. The most important ladders are game-entertainment-interest-fun and enjoyment, games-entertainment-interest-happiness, time/alarm-daily life support-convenience-vibrant life, calendar/memo/diary-daily life support-convenience-vibrant life, and map navigation-daily life support-convenience-vibrant life.

Figure 2 shows that the HVM for low-involvement users is similar to that for high-involvement users. This finding indicates that involvement is not a key factor for smart phone users. Smart phone users derive convenience value from the attributes on offer, such as voice call/video call. For smart phone game players, the values derived by these users are fun, enjoyment, and happiness.

Figure 3 and Figure 4 show that the HVM of portal site users is simpler than that of smart phone users. However, a focus on convenience exists at the psychological consequences stage.

Figure 5 and Figure 6 show that the HVM of SNS users is different from that of portal sites or smart phone users. The connections between each step are spread diversely. Many ladders are linked to the "warm relationship with others" value. 
Table 17. Implication matrix of relationships of attributes and functional consequences for SNS users by involvement.

\begin{tabular}{ccccccc}
\hline \multirow{2}{*}{ Item } & \multicolumn{4}{c}{ Involvement } & \multirow{2}{*}{ Total } \\
\cline { 2 - 5 } My account/scheduling-daily life support & \multicolumn{3}{c}{ Low } & \multicolumn{2}{c}{ High } & \\
Online social networking-social relationships & 168 & $(9.4 \%)$ & 170 & $(10.0 \%)$ & 338 & $(9.7 \%)$ \\
Friends/institution introduction-social relationships & 178 & $(9.9 \%)$ & 165 & $(9.7 \%)$ & 343 & $(9.8 \%)$ \\
Friends/institution searching-social relationships & 132 & $(7.4 \%)$ & 146 & $(8.6 \%)$ & 278 & $(8.0 \%)$ \\
Aggregation of news-speedy information retrieval/content acquisition & 97 & $(5.4 \%)$ & 95 & $(5.6 \%)$ & 192 & $(5.5 \%)$ \\
Mentionor comments-social relationships & 115 & $(6.4 \%)$ & 120 & $(7.0 \%)$ & 235 & $(6.7 \%)$ \\
Mention or comments-mutual communication & 106 & $(5.9 \%)$ & 90 & $(5.3 \%)$ & 196 & $(5.6 \%)$ \\
Sns game-entertainment & 168 & $(9.4 \%)$ & 156 & $(9.2 \%)$ & 324 & $(9.3 \%)$ \\
E-commerce-daily life support & 142 & $(7.9 \%)$ & 136 & $(8.0 \%)$ & 278 & $(8.0 \%)$ \\
Send gifts and items-social relationship & 149 & $(8.3 \%)$ & 133 & $(7.8 \%)$ & 282 & $(8.1 \%)$ \\
Total & 1790 & $(100.0 \%)$ & 1703 & $(100.0 \%)$ & 3493 & $(100.0 \%)$ \\
\hline
\end{tabular}

Table 18. Implication matrix of relationships between psychological consequences and functional consequences for SNS Users by Involvement.

\begin{tabular}{ccccccc}
\hline \multirow{2}{*}{ Items } & \multicolumn{3}{c}{ Involvement } & \multicolumn{3}{c}{ Total } \\
\cline { 2 - 5 } & \multicolumn{3}{c}{ Low } & \multicolumn{2}{c}{ High } & \\
Daily life support-convenience & 88 & $(7.7 \%)$ & 93 & $(7.9 \%)$ & 181 & $(7.8 \%)$ \\
Self-expression-self satisfaction & 103 & $(9.0 \%)$ & 111 & $(9.4 \%)$ & 214 & $(9.2 \%)$ \\
Social relationships-well-rounded social life & 83 & $(7.2 \%)$ & 72 & $(6.1 \%)$ & 155 & $(6.7 \%)$ \\
Mutual communication-well-rounded social life & 129 & $(11.3 \%)$ & 136 & $(11.5 \%)$ & 265 & $(11.4 \%)$ \\
Speedy information retrieval/contents acquisition-well-rounded social life. & 104 & $(9.1 \%)$ & 117 & $(9.9 \%)$ & 221 & $(9.5 \%)$ \\
Speedy information retrieval/contents acquisition-empathy & 101 & $(8.8 \%)$ & 83 & $(7.0 \%)$ & 184 & $(7.9 \%)$ \\
A variety of information/contents acquisition-convenience & 109 & $(9.5 \%)$ & 116 & $(9.8 \%)$ & 225 & $(9.7 \%)$ \\
Information/contents individual storage-convenience & 108 & $(9.4 \%)$ & 107 & $(9.1 \%)$ & 215 & $(9.3 \%)$ \\
Sharing info/contents-convenience & 94 & $(8.2 \%)$ & 107 & $(9.1 \%)$ & 201 & $(8.6 \%)$ \\
Work (mobile office)-convenience & 74 & $(6.5 \%)$ & 83 & $(7.0 \%)$ & 157 & $(6.8 \%)$ \\
Total & 1146 & $(100.0 \%)$ & 1178 & $(100.0 \%)$ & 2324 & $(100.0 \%)$ \\
\hline
\end{tabular}

Table 19. Implication matrix of relationships between psychological consequences and value for SNS users by involvement.

\begin{tabular}{ccccccc}
\hline \multirow{2}{*}{ Items } & \multicolumn{4}{c}{ Involvement } & \multicolumn{2}{c}{ Total } \\
\cline { 2 - 5 } & \multicolumn{3}{c}{ Low } & \multicolumn{2}{c}{ High } & \\
Self-satisfaction-self realisation & 88 & $(9.7 \%)$ & 90 & $(10.4 \%)$ & 178 & $(10.0 \%)$ \\
Self-pride-self accomplishment & 88 & $(9.7 \%)$ & 75 & $(8.6 \%)$ & 163 & $(9.2 \%)$ \\
Self-cultivation-self realisation & 65 & $(7.1 \%)$ & 78 & $(9.0 \%)$ & 143 & $(8.0 \%)$ \\
Self-cultivation-self accomplishment & 82 & $(9.0 \%)$ & 60 & $(6.9 \%)$ & 142 & $(8.0 \%)$ \\
Desire to possess-warm relationship with others & 116 & $(12.7 \%)$ & 118 & $(13.6 \%)$ & 234 & $(13.2 \%)$ \\
Well-rounded social life-friendly relationship with others & 121 & $(13.3 \%)$ & 121 & $(13.9 \%)$ & 242 & $(13.6 \%)$ \\
Trust-warm relationship with others & 81 & $(8.9 \%)$ & 79 & $(9.1 \%)$ & 160 & $(9.0 \%)$ \\
Preference taste-happiness & 89 & $(9.8 \%)$ & 67 & $(7.7 \%)$ & 156 & $(8.8 \%)$ \\
Interest-fun and enjoyment & 107 & $(11.8 \%)$ & 115 & $(13.2 \%)$ & 222 & $(12.5 \%)$ \\
Interest-happiness & 73 & $(8.0 \%)$ & 66 & $(7.6 \%)$ & 139 & $(7.8 \%)$ \\
Total & 910 & $(100 \%)$ & 869 & $(100 \%)$ & 1779 & $(100 \%)$ \\
\hline
\end{tabular}




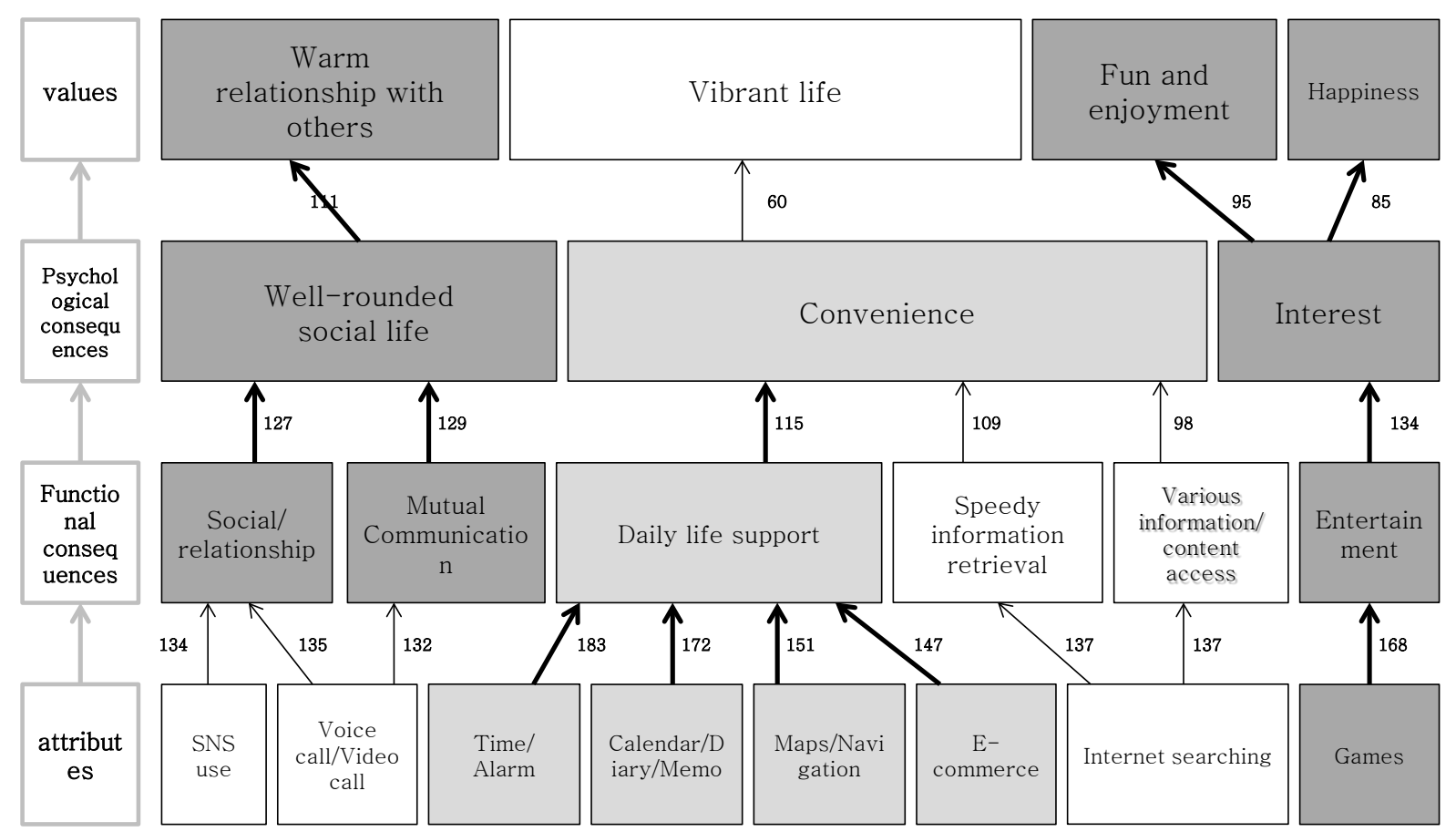

Figure 1. HVM for high-involvement smart phone users.

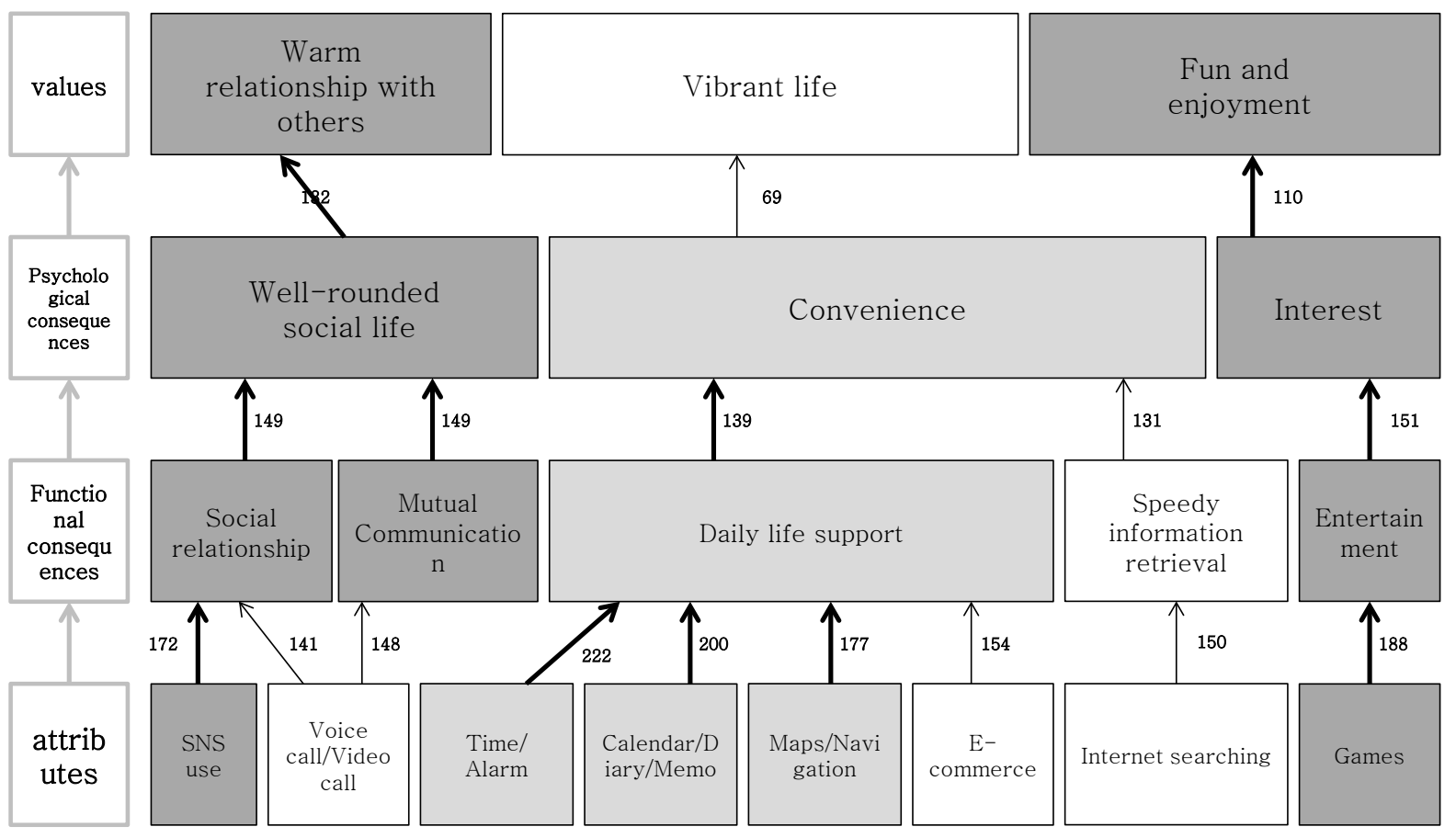

Figure 2. HVM for low-involvement smart phone users.

\section{Discussions}

In this study, we derived the HVMs of smart phones, portal sites, and SNSs depending on user involvement based on the concept of involvement and means-chain theory. We identified differences in values among users in the high-involvement group and users in the low-involvement group for all media types.

The most important value for the high-involvement group is self-accomplishment. The members of the high- 


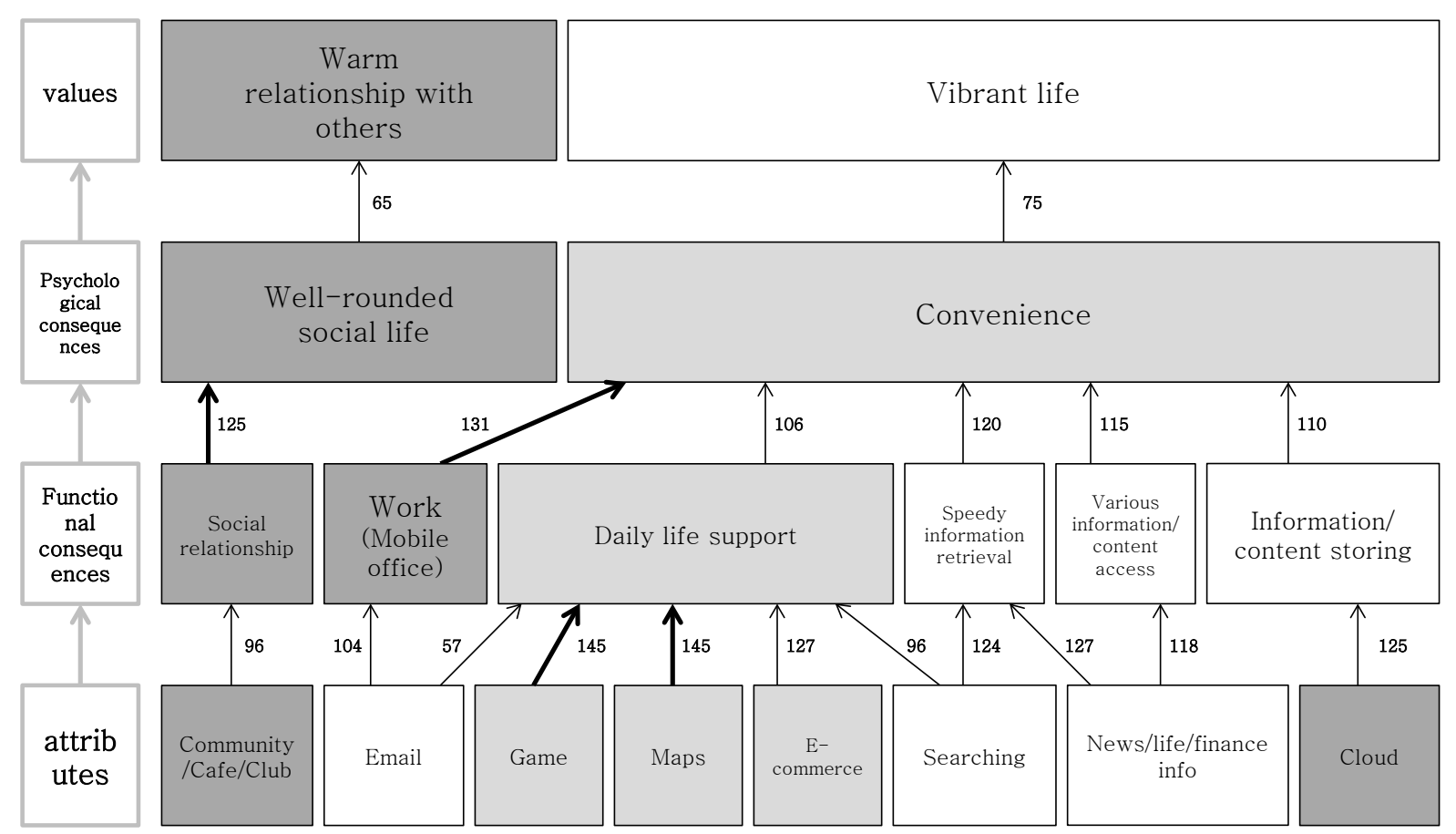

Figure 3. HVM for high-involvement portal site users.

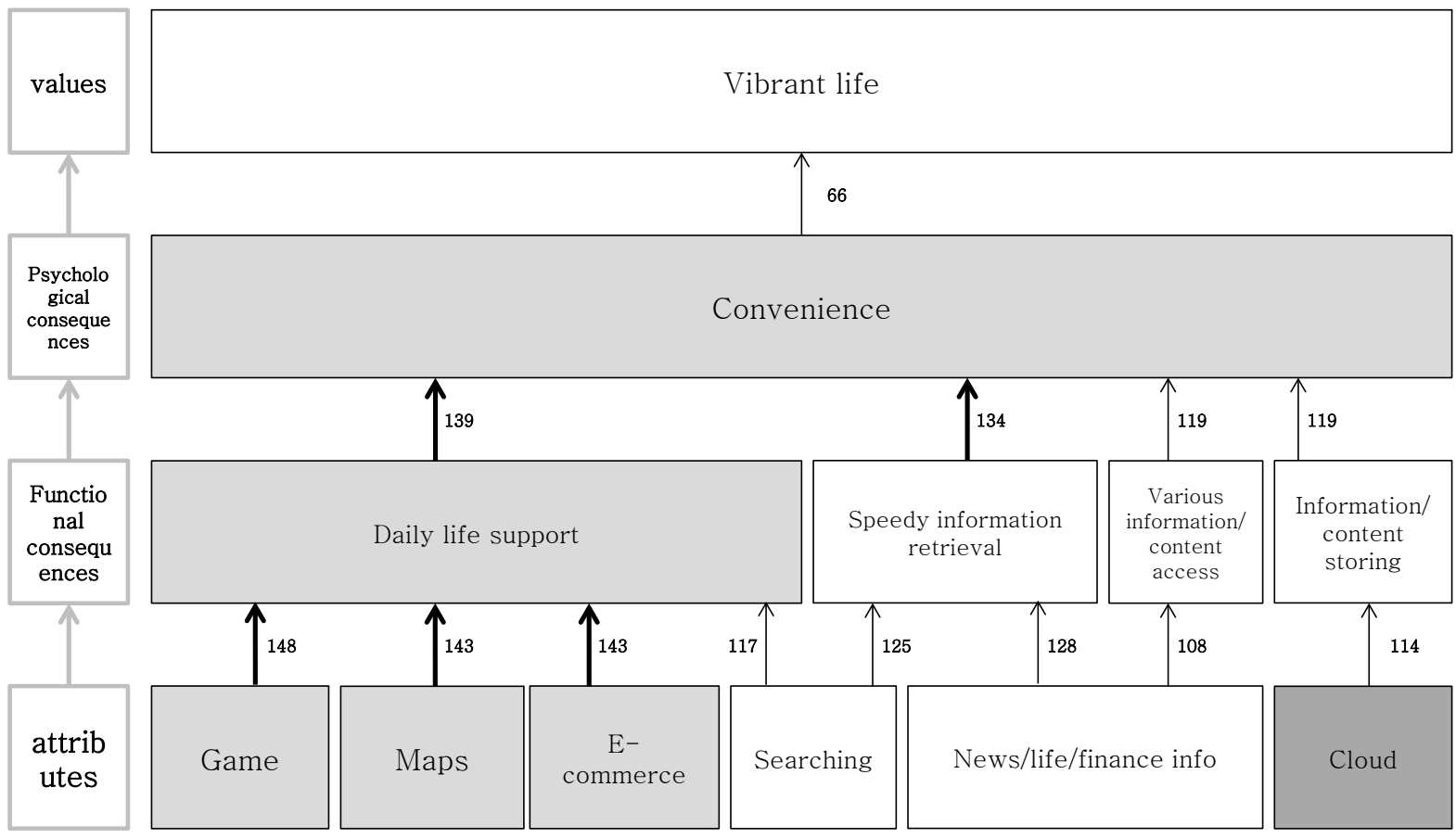

Figure 4. HVM for low-involvement portal site users.

involvement group tend to be very active users who use media as a means to achieve their goals. The most important values for the low-involvement group are self-realization and friendly relationships with others. In addition, high-and low-involvement HVMs are apparently similar with regard to motivation and behavior of media users. However, the perceptions of attributes, consequences, and utilization of media show differences depending on involvement. 


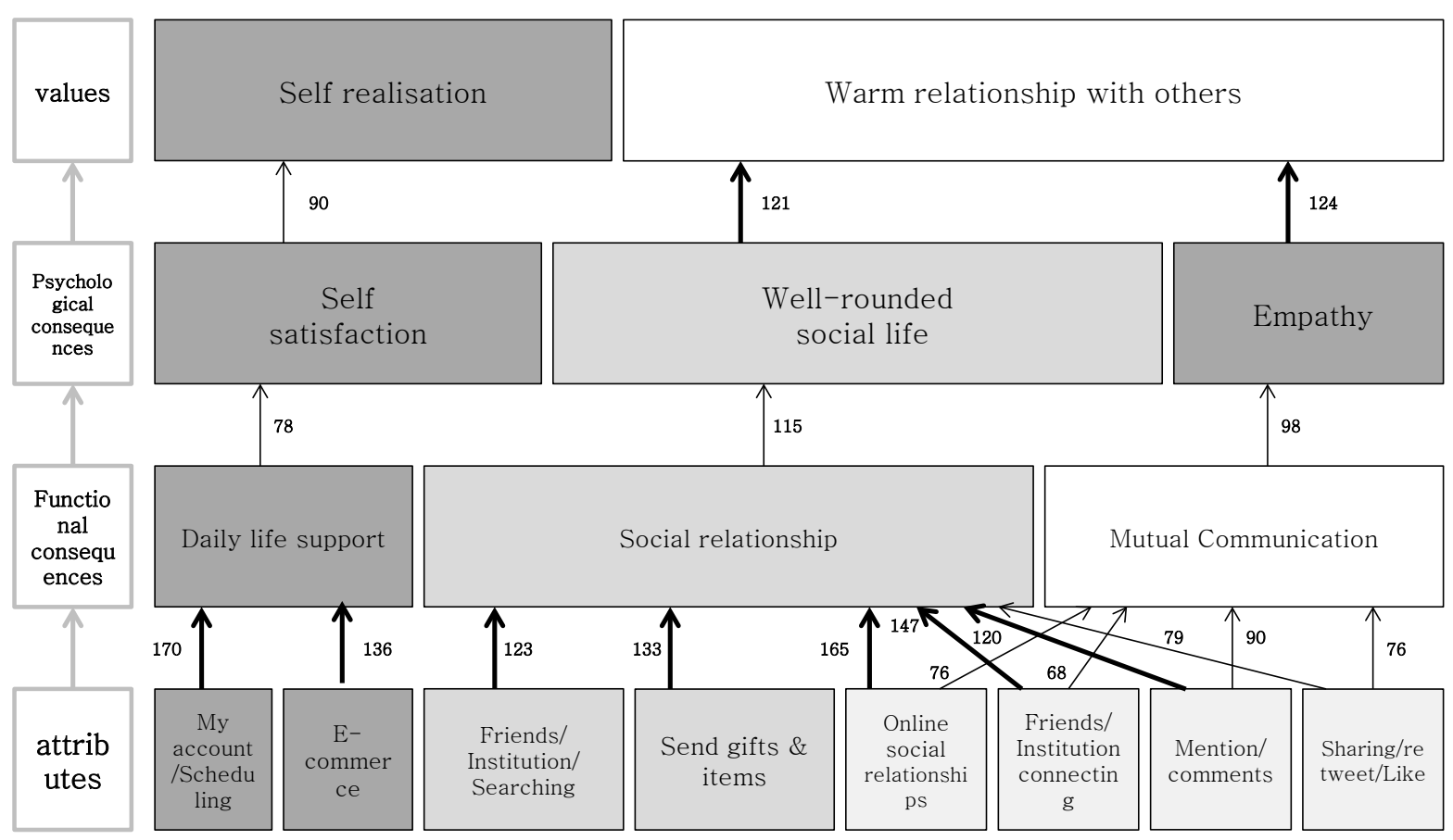

Figure 5. HVM for high-involvement SNS users.

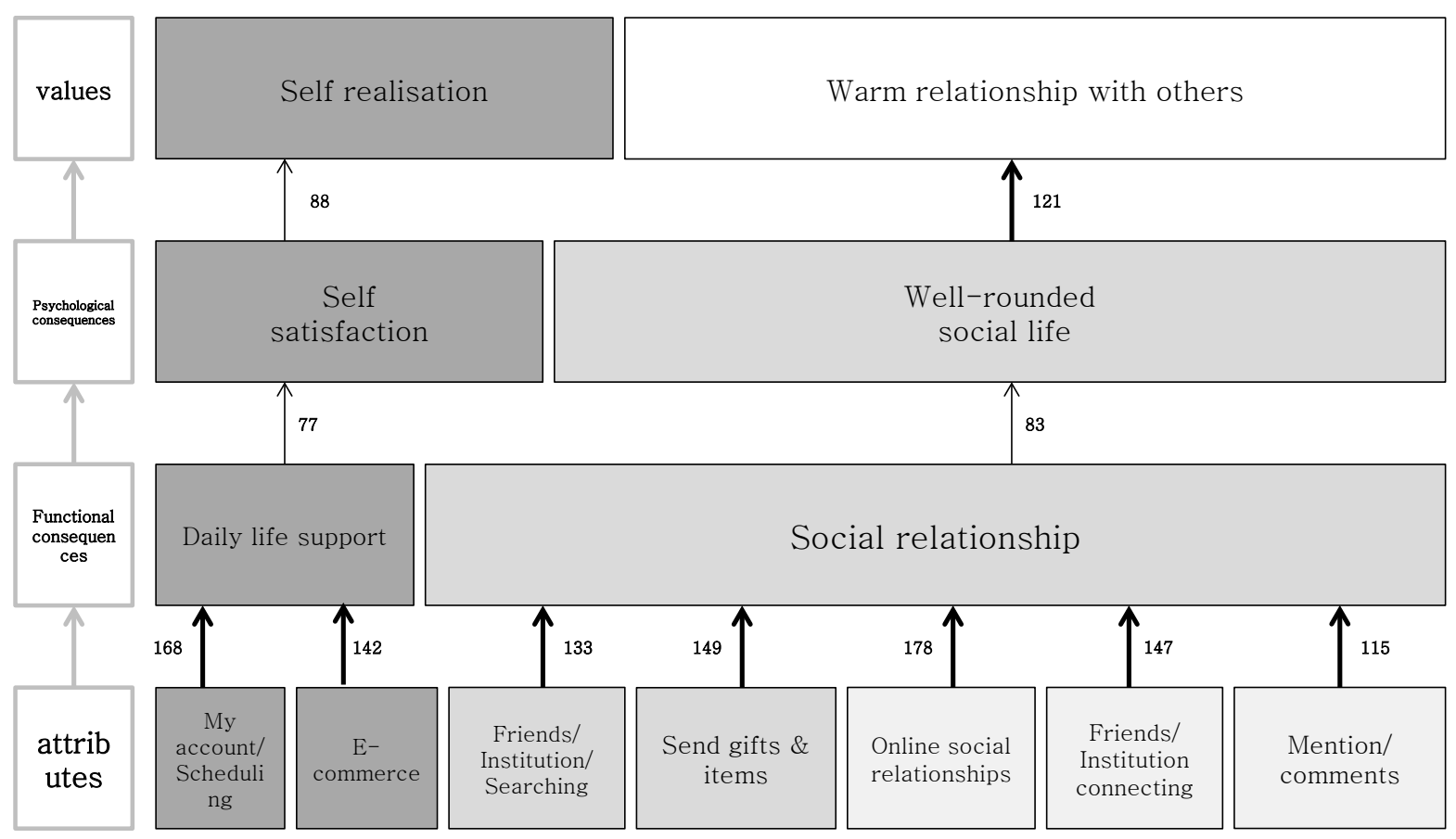

Figure 6. HVM for low-involvement SNS users.

The implication of the research findings is that different usage values exist in cyber media. The result shows that the basic values for smart phone users are social life support, entertainment, social relationship, and interaction communication. In comparison, the important values for portal site users are quick information acquisition, supporting daily life, and a variety of information/content acquisition and entertainment. The HVMs of smart phones, portal sites, and SNSs were compared with one another, and the result shows that they have different value chains. 
The final values of users are also influenced by the characteristics and functional strength of media. New communication devices constantly attempt to meet the basic human desire for communication because desire drives the demand for new social smart devices.

\section{References}

Baudrillard, J. (1970). The Consumer Society: Myths and Structures. Theory, Culture \& Society. New York: Nottingham University.

Celsi, R. L., \& Olson, J. C. (1988). The Role of Involvement in Attention and Comprehension Processes. Journal of Consumer Research, 15, 210-224. http://dx.doi.org/10.1086/209158

Christensen, G. L., \& Olson, J. C. (2001). Involved with What? The Impact of Heterogeneity in Goal Hierarchies on High Enduring Involvement. Advances in Consumer Research, 28, 392.

Grunert, K., \& Grunert, S. (1995). Measuring Subjective Meaning Structures by the Laddering Method: Theoretical Considerations and Methodological Problems. International Journal of Research in Marketing, 12, 209-225. http://dx.doi.org/10.1016/0167-8116(95)00022-T

Gutman, J. (1981). A Means-End Model for Facilitating Analysis of Product Markets Based on Consumer Judgement. Advances in Consumer Research, 8, 116-121.

Gutman, J. (1982). A means-End Chain Model based On Consumer Categorisation Processes. Journal of Marketing, 46, 60-72. http://dx.doi.org/10.2307/3203341

Huber, F., Beckmann, S. C., \& Herrmann, A. (2004). Means-End Analysis: Does the Affective State Influence Information Processing Style? Psychology \& Marketing, 21, 715-737. http://dx.doi.org/10.1002/mar.20026

Maslow, A. (1943). A Theory of Human Motivation. Psychological Review, 50, 370-396. http://dx.doi.org/10.1037/h0054346

Peter, J. P., \& Olson, J. C. (2005). Consumer Behaviour and Marketing Strategy (7th ed). New York: McGraw-Hill/Irwin.

Petty, R. E., \& Cacioppo, J. T. (1986). Communication and Persuasion: Central and Peripheral Routes to Attitude Change. New York: Springer-Verlag. http://dx.doi.org/10.1007/978-1-4612-4964-1

ter Hofstede, F., Audenaert, A., Steenkamp, J. E. M., \& Wedel, M. (1998). An Investigation into the Association Pattern Technique as a Quantitative Approach to Measuring Means-End Chain. International Journal of Research in Marketing, 15, 37-50. http://dx.doi.org/10.1016/S0167-8116(97)00029-3

Woodruff, R. B., \& Gardial, S. F. (1996). Know Your Customer: New Approaches to Understanding Customer Value and Satisfaction. Cambridge, MA: Blackwell Publishers, Inc.

Zaichkowsky, J. L. (1985). Measuring the Involvement Construct. Journal of Consumer Research, 12, 341-352. http://dx.doi.org/10.1300/J047v06n04 05 